\title{
INVESTIGATIONAL METHODS FOR THE MODELING OF INFILTRATION PROCESS IN THE SOIL AND THE ESTIMATING THE SOIL HYDRODYNAMIC PARAMETERS
}

\author{
M. HACHIMI ${ }^{1}$, A. MASLOUHI ${ }^{1}$, K. TAMOH $^{1}$, H. QANZA $^{1}$, \\ 1. Interdisciplinary Laboratory for Natural Resources and Environment \\ Department of Physics, Faculty of Sciences, Ibn Tofail University, B.P 242, 14000 Kenitra, Morocco. \\ Email corresponding author: mustapha_hachimi@yahoo.fr
}

\begin{abstract}
Unsaturated zone in the soil generally plays an important role in the transfer of water and pollutants in the underground environment. In this context, the determination of the hydrodynamic parameters constitutes an essential step for any study of transfers of water and solutes in the unsaturated zone. The purpose of this article is the estimation the soil hydrodynamic parameters by the direct method and the inverse method, from the infiltration data by using the disc infiltrometer in the soil the basin Loukkos located in northern Morocco. Our results the numerical modeling reproduced correctly the experimental measurements. These results show that the inverse method remains a robust and accurate method for determining the soil hydrodynamic parameters compared to other conventional methods.
\end{abstract}

Keywords: Unsaturated soil, Infiltration, Hydraulic parameters, modeling, Loukkos Basin

\section{Introduction}

In soil science, the modeling of water flows and the transport of pollutants in a porous medium is an active domain in various fields, among which agronomy and environmental protection and especially the contamination of groundwater by fertilizers and pesticides. The study of the hydrological processes of the soil, in particular, to model the transport of water and solutes in the unsaturated zone, requires the knowledge of the soil hydrodynamic parameters [8]. These soil hydrodynamic properties are defined as follows: the residual water content $\theta_{r}\left(\mathrm{~cm}^{3} . \mathrm{cm}^{-3}\right)$, saturation water content $\theta_{s}\left(\mathrm{~cm}^{3} . \mathrm{cm}^{-3}\right)$, saturation hydraulic conductivity $K_{s}$ $\left(m . s^{-1}\right)$ and shape parameters $(\alpha, n)$ van Genuchten model used. Them in-situ estimation presents difficulties related to several causes, mainly spatial variability, inhomogeneity of the soil, and some errors related to the nature of field experiments $[1 ; 4]$. In this context, we address the inverse problem for estimating the hydrodynamic parameters to facilitate the modeling of water transfer in the soil up to the groundwater.

The aim of this study is to obtain the most accurate characterization of the hydrodynamic properties $\left(\theta_{r}, \theta_{s}, \alpha\right.$, $n, K_{s}$ ) of R'mel and M'risa soils with a minimum of field data. The characterization was performed by two different methods: direct method and inverse method. The infiltration experiments were carried out in situ, by using the disc infiltrometer [7], at two experimental stations, R'mel and M'risa, in the Loukkos Basin located in northern Morocco.

\section{Material and methods}

\subsection{Field measurement}

The experimental study constitutes an essential basis of the work exposed in this article. It relies a series of measurements obtained in July 2013 and in September 2015. The selected sites are R'mel and M'risa from the Loukkos Basin located in northern Morocco. We carry out a granulometric analysis which demonstrates the texture and the soil structure of two sites studied (Table 1). This table shows that the R'mel soil is totally sandy and the M'risa soil is clay-loam. A plot of $10 \mathrm{~m}^{2}$ was chosen in the two experimental stations R'mel and M'risa. Before starting the experiment, soil samples were taken using a metal cylinder of volume $250 \mathrm{~cm}^{3}$ to calculate the initial water content $\theta_{i}$, the dry density $\rho_{d}$ and the soil porosity $P$ (Table 1). The device used during the infiltration experiments is the disc infiltrometer, figure 1, which is an instrument commonly used for the in-situ estimation of the soil hydraulic properties [1].

Table 1. Texture and characteristics of studied soils

\begin{tabular}{lllc}
\hline & Rmel soil & & M'risa soil \\
\cline { 1 - 1 } Texture class & Sandy & Loam-Clay \\
Density $\rho_{\mathrm{d}}\left(10^{3} \mathrm{~kg} \cdot \mathrm{m}^{-3}\right)$ & & 1,493 & \\
Porosity $\mathrm{P}\left(\mathrm{cm}^{3} \cdot \mathrm{cm}^{-3}\right)$ & 0,421 & 0,411 \\
Initial water content & 0,022 & 0,185 \\
$\theta_{\mathrm{i}}\left(\mathrm{cm}^{3} \cdot \mathrm{cm}^{-3}\right)$ & & \\
\hline
\end{tabular}




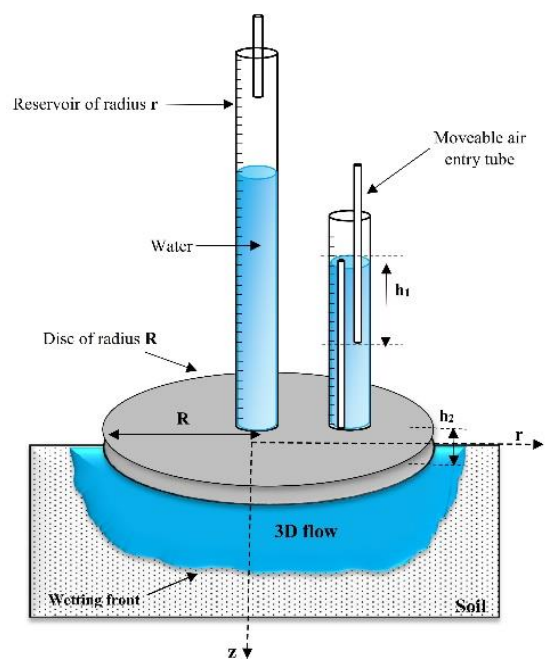

Figure 1: Disc infiltrometer and wetting front profile

\subsection{Direct method}

Hydrodynamic characterization of soils, by the direct method, requires the determination of functions the water retention $\theta(h)$ and the hydraulic conductivity $K(\theta)$. For the retention function we used the Van-Genuchten model (1980):

$$
\theta(h)=\theta_{r}+\left(\theta_{s}-\theta_{r}\right)\left(1+|\alpha h|^{n}\right)^{-m}
$$

And the Brooks-Corry model (1964) for the hydraulic conductivity function:

$$
K(\theta)=K_{s}\left(\frac{\theta-\theta_{r}}{\theta_{s}-\theta_{r}}\right)^{\eta}
$$

Where, $\theta_{r}\left(\mathrm{~cm}^{3} \cdot \mathrm{cm}^{-3}\right)$ and $\theta_{s}\left(\mathrm{~cm}^{3} \cdot \mathrm{cm}^{-3}\right)$ are respectively the residual water contents and saturated. $K_{s}\left(m \cdot s^{-1}\right)$ is the saturated hydraulic conductivity. $n(-), m(-), \eta(-)$ and $\alpha$ $\left(\mathrm{cm}^{-1}\right)$ are shape parameters of the models. With, $m=1-2 / n$ and $\eta=2 / m n+3$.

The direct method, for estimating the soil hydrodynamic parameters, is based on the manual calibration of the functions $\theta(h)$ and $K(\theta)$ on the experimental data obtained previously. Among the disadvantages of this method, is that it requires several measurements, take a lot of time-consuming and is expensive.

\subsection{Inverse method}

Manual calibration is often cumbersome and time-consuming. This procedure can be automated by the inverse approach. Estimation of the hydrodynamic parameters $\beta=\left(\theta_{r}, \theta_{s}, n, \alpha, K_{s}\right)$ by this method is based on the choice of the infiltration equation. Many empirical expressions have been proposed to describe the infiltration of water from the disc infiltrometer. Among these expressions, we choose that of Talsma and Parlange [9].

The generalized form of this equation is as follows For $h_{0} \leq 0$ :

$$
I(t)=K_{S} \cdot t-\left(\theta_{s}-\theta_{0}\right) \cdot\left(-\frac{C_{p}}{2} h_{g}\right) \cdot\left[\exp \left(-\frac{I-K_{0} \cdot t}{\left(\theta_{s}-\theta_{0}\right) \cdot\left(-\frac{C_{p}}{2} h_{g}\right)}\right)-1\right]
$$

With, $h_{g}(\mathrm{~cm})=1 / \alpha$ is a normalization parameter. $C_{p}$ is a parameter expressed using the Gamma function $\Gamma$ by:

$$
C_{p}=\Gamma\left(1+\frac{1}{n}\right)\left\{\frac{\Gamma\left(m \cdot \eta-\frac{1}{n}\right)}{\Gamma(m \cdot \eta)}+\frac{\Gamma\left(m \cdot \eta+m-\frac{1}{n}\right)}{\Gamma(m \cdot \eta+m)}\right\}
$$

The inverse approach is based on the minimization of the objective function $\Phi(\beta)$, representing the quadratic differences between the measured and simulated values, defined by:

$$
\Phi_{I}(\beta)=\sum_{i=1}^{N} \lambda_{I}\left(I^{\exp }\left(t_{i}\right)-I^{\operatorname{sim}}\left(t_{i}, \beta\right)\right)^{2}
$$

\subsection{Approximate profiles of infiltration}

The hydrodynamic parameters, thus estimated from the cumulative infiltration curve $I(t)$, make it possible to calculate the vertical infiltration front (or the water profile) in the soil. This solution makes it possible to calculate the infiltration front in a simple and fast way and with precision. The water profiles, $z(\theta, t)$, are obtained by equation:

$$
z(\theta, t)=\left[I(t)-K_{0} \cdot t\right] \frac{\left(\left(\theta^{*}\right)^{-\frac{1}{\tau}}-1\right)^{\frac{1}{\tau}}}{\theta_{s} \int_{\theta_{i}^{*}}^{\theta_{s}^{*}}\left(\left(\theta^{*}\right)^{-\frac{1}{\tau}}-1\right)^{\frac{1}{\tau}} d \theta^{*}}
$$

\section{Results and discussions}

\subsection{Direct method}

To estimate the soil hydrodynamic parameters by the direct method, we made a number of in situ measurements of the water retention curve $h(\theta)$ and hydraulic conductivity $K(\theta)$. To do this, we performed infiltration experiments at different locations and with different pressures $h_{0}$ between $-20 \mathrm{~cm}$ and $0 \mathrm{~cm}\left(-20 \leq h_{0} \leq 0 \mathrm{~cm}\right)$ on two experimental stations R'mel and M'risa. In addition, the measurements points were adjusted by van Genuchten (Eq.1) and Brooks-Corey (Eq.2) models to determine the desired hydrodynamic parameters. Figures 2 shows the results of this adjustment for both R'mel and M'risa soils. Table 2 summarizes the hydrodynamic parameters that results from this adjustment. However, such an order of magnitude of error remains acceptable for field measurements, given the other sources of errors affecting the computation of the volume water content $\theta$ and the hydraulic conductivity $K$ from the infiltration tests.
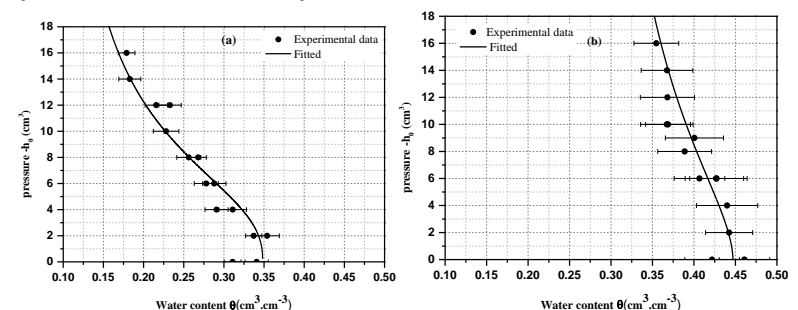

Figure 2: Fitting of the retention curve $h(\theta)$ : (a) R'mel soil and (b) M'risa soil. 
Table 2. Estimated values of the hydrodynamic parameters by the direct method.

\begin{tabular}{|c|c|c|}
\hline & Rmel soil & M'risa soil \\
\hline Parameter & Estimted value & Estimted value \\
\hline$\theta_{\mathrm{r}}\left(\mathrm{cm}^{3} \cdot \mathrm{cm}^{-3}\right)$ & 0.085 & 0.231 \\
\hline$\theta_{\mathrm{s}}\left(\mathrm{cm}^{3} \cdot \mathrm{cm}^{-3}\right)$ & 0.348 & 0.447 \\
\hline$\alpha\left(\mathrm{cm}^{-1}\right)$ & 0.127 & 0.102 \\
\hline n (-) & 2.479 & 1.740 \\
\hline $\mathrm{K}_{\mathrm{s}}\left(\mathrm{m} \cdot \mathrm{s}^{-1}\right)$ & $5.281 \times 10^{-5}$ & $1.970 \times 10^{-6}$ \\
\hline$\eta(-)$ & 4.295 & 3.437 \\
\hline
\end{tabular}

\subsection{Inverse method}

To overcome the difficulties encountered in the direct method, we then applied the inverse method on the two selected sites. Estimation of the hydrodynamic parameters by the inverse approach is based on the combination of the cumulative infiltration data $I(t)$, (Eq.3), and the minimization of the objective function (Eq.5) by the optimization Levenberg-Marquardt algorithm. Table 3 presents the values of the hydrodynamic parameters $\left(\theta_{s}, n, \alpha, K_{s}\right)$ obtained by this optimization for the two soils. Note that the parameter $n$ is calculated from the expression of $C_{p}$ (Eq. 4). The parameters obtained are generally of the same order of magnitude for the two pressures $h_{0}=-6 \mathrm{~cm}$ and $h_{0}=-10$ $\mathrm{cm}$. Such an order of magnitude of errors remains acceptable for in situ measurements.

Table 3. Parameters values estimated by inverse method.

\begin{tabular}{lll|ll}
\hline & \multicolumn{2}{c|}{ Rmel soil } & \multicolumn{2}{c}{ M'risa soil } \\
\cline { 2 - 5 } & $\mathrm{h}_{0}=-6$ & $\mathrm{~h}_{0}=-10$ & $\mathrm{~h}_{0}=-6$ & $\mathrm{~h}_{0}=-10$ \\
& $\mathrm{~cm}$ & $\mathrm{~cm}$ & $\mathrm{~cm}$ & $\mathrm{~cm}$ \\
\hline$\theta_{\mathrm{s}}\left(\mathrm{cm}^{3} \cdot \mathrm{cm}^{-3}\right)$ & 0.377 & 0.413 & 0.4835 & 0.4672 \\
$\mathrm{~h}_{\mathrm{g}}(\mathrm{cm})$ & -6.52 & -7.4 & -9.699 & -10.741 \\
$\alpha\left(\mathrm{cm}^{-1}\right)$ & 0.153 & 0.1351 & 0.103 & 0.0947 \\
$\mathrm{~K}_{\mathrm{s}}\left(\mathrm{m}_{\mathrm{s}} \mathrm{s}^{-1}\right)$ & $2.3 \times 10^{-5}$ & $2.84 \times 10^{-5}$ & $1.63 \times 10^{-6}$ & $1.253 \times 10^{-6}$ \\
$\mathrm{C}_{\mathrm{p}}(\mathrm{cm})$ & 1.92 & 1.8 & 3.64 & 3.89 \\
$\mathrm{n}(-)$ & 2.490 & 2.675 & 1.942 & 1.922 \\
\hline
\end{tabular}

\subsection{Evolution of infiltration profiles}

In this section, we calculated the vertical infiltration front in the soil, from the hydrodynamic parameters, thus estimated, by using a new analytical expression (Eq.6). This solution makes it possible to calculate the infiltration front in a simple and fast way and with precision. Figure 3 shows the evolution of the infiltration profiles obtained at different times for the two soils studied. The sandy soils (R'mel soil) shows that the profiles are well distinguished and that the infiltration front evolves dramatically, against clay-loam soils (M'risa soil), knowing that both soils are subjected to the same pressure $h_{0}=-6 \mathrm{~cm}$.
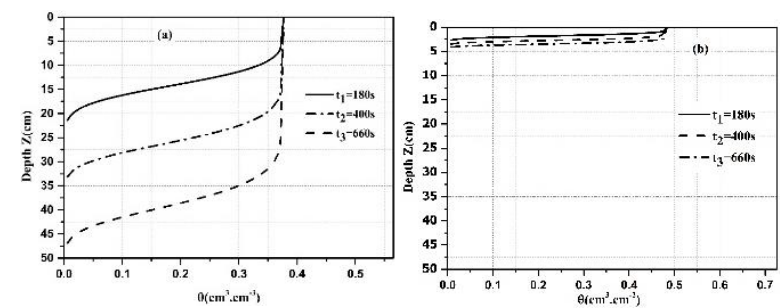

Figure 9: Infiltration profiles calculated at $h_{0}=-6 \mathrm{~cm}$ :

(a) R'mel soil and (b) M'risa soil

\section{Conclusion}

Hydraulic parameters of unsaturated soil are essential for many applications in the porous medium. The study presented in this paper concerns the in-situ identification of the hydrodynamic parameters $\left(\theta_{r}, \theta_{s}, n, \alpha\right.$, $K_{s}$ ) of the R'mel and M'risa soils of the Loukkos Basin, located in northern Morocco. At first, we performed the infiltration tests, by using the disc infiltrometer. In a second step, the hydraulic parameters were estimated by the direct method and the inverse method, from the infiltration data. The results obtained shows that the inverse method is less restrictive and relevant for the hydrodynamic characterization of soils.

The technique developed as part of this work may be useful for scientific researchers interested in studying the transfer of water through the unsaturated zone and towards the aquifer.

\section{Références}

[1] Hachimi M., and A. Maslouhi. 2016. "Hydrodynamic characterization using the disc infiltrometer of Loukkos soils (Morocco)". Journal of Materials and Environmental Science, 7 (9): 3300-3312.

[2] Hmimou, A., A., Maslouhi, K. Tamoh, and L. Candela. 2014. "Experimental monitoring and numerical study of pesticide (carbofuran) transfer in an agricultural soil at a field site”. Comptes Rendus Geoscience 346(9): 255261.

[3] Marquardt D. 1963. "An algorithm for Leastsquares estimation of nonlinear parameters". Journal for the society of Industrial and applied Mathematics 11, 431- 441.

[4] Perroux, K. M., and White, I. 1988. "Designs for disc permeameters". Soil Science Society of America Journal, 52(5), 1205-1215.

[5] Talsma, T., and Parlange J.-Y. 1972. "Onedimensional vertical infiltration". Soil Research, 10(2), 143-150.

[6] Tamoh K. and Maslouhi A. 2003. "Mesures in situ par infiltrométrie des propriétés hydrodynamiques des sols de Mnasra (Maroc)". Comptes Rendus Géoscience, 336, (6), 535-545. 